THEORIA ET HISTORIA SCIENTIARUM, VOL. VIII, $\mathrm{N}^{\circ} 1$

Ed. Nicolaus Copernicus University 2008

Sergio E. Chaigneau, Lawrence W. Barsalou

\title{
The Role of Function in Categories
}

\begin{abstract}
In the psychological literatures on function, four issues have been important: (1) whether function can be a core property of the concepts that represent categories, (2) whether categories based primarily on function provide support for inductive inference, (3) whether functions guide object naming in children, (4) whether function is best understood as affordances or as design history. In these debates, function is often viewed as an independent unitary property that can exist independently of an object's physical structure. We propose instead that function is a complex relational system that links physical structure, settings, action, and design history. Furthermore we show that viewing function this way resolves discrepancies in the empirical literatures that address it. In particular we find that function achieves its greatest importance when subjects understand the complex relational systems that underlie it. When subjects do not understand these systems, function's role in classification, inductive inference, and naming decreases. Viewing function as a complex relational system highlights the need for future explorations into its conceptual structure.
\end{abstract}

At least from a layperson's point of view, an object's function-the use to which people put it - is a central aspect of the object's conceptualization. Who would not include used to put screws in place when explaining what a screwdriver is? Researchers in categorization, however, have not always endorsed this position. In fact, much controversy surrounds the role of function in categories. The central question is whether function plays a major role in the concepts that represent categories, or whether it is peripheral.

Surprisingly, for all the research performed on the topic, the conceptual structure of function remains largely unexplored (but see Wright 1973). Typically function is treated as a simple unitary property that can be predicated of an object independently of its other properties, such as its physical structure and 
contexts of use. Contrary to this view, we propose that function is a complex relational system. This is not a new theme, given all of the work that demonstrates the importance of relational systems in cognition, including Gentner and Markman (1997), Markman and Gentner (1997), Holyoak and Thagard (1997), Pearl (2000), Sloman, Love, and Ahn (1998), Ahn (1999), and Barsalou (1992). In the spirit of this previous work, we will argue that an object's function emerges from a relational system that links its physical structure with its use, background settings, and design history.

In this article we show how understanding function as a relational system is helpful for interpreting empirical results, resolving discrepancies between findings, and reconciling contradictory views on the nature of function. To demonstrate these points, we review and analyze the literatures on four issues: (1) whether function can be a core property of concepts, (2) whether a category organized around function provides support for inductive inference, (3) whether function can guide object naming in children, and (4) whether function should be understood as affordances or as design history.

\section{Problem 1: Can Function be a Core Property of Concepts?}

One central concern of categorization researchers has been whether a given property can be necessary and sufficient for category membership. Necessary and sufficient properties - technically called core properties-allow subjects to decide definitively whether an exemplar belongs to a category. For example, if feeding its offspring with milk were a core property of mammal, then when given an unknown animal, verifying the presence of a single property determines its category membership.

Theories of concepts disagree about whether core properties exist. On one hand, exemplar and prototype theories assume they do not. Exemplar theories hold that category coherence arises out of similarity, not from the existence of core properties (e.g., Estes 1986; Hampton 1979, 1995; Heit 1992; Hintzman 1986; Medin \& Schaffer 1978; Nosofsky 1986, 1991). Prototype theories make the same assumption (e.g., Rosch 1975; Rosch \& Mervis 1975; Rosch, Simpson \& Miller 1976; Smith \& Sloman 1994). For example, on finding a new chair subjects classify it accordingly because it resembles other chairs encountered, not because it has necessary and sufficient chair properties. On the other hand, theory-theories contend that similarity is too unconstrained to be considered the basis of categorization (e.g., Medin \& Ortony 1989; Smith \& Medin 1981). Instead the theory-based approach argues that properties are often causally related to each other (e.g., feathers and being able to fly). As a result, the correct way to understand concepts is as theories about relations between core and surface 
properties. The core properties that underlie categories reside at the level of the theory and are not implicated directly in object recognition, which often relies more on surface similarity.

The issue of whether functions can be core properties is highly contested. A number of studies suggest that function can indeed be a core property, consistent with the theorytheory. If function is a core property, it means that knowing what an object is used for should be sufficient to classify it accurately (e.g., knowing that something is used to put screws in place should be sufficient to categorize it as a screwdriver). As described next, Barton and Komatsu (1989) and Keil (1989) provide support for this view.

Barton and Komatsu (1989) performed two experiments. In Experiment 1, subjects were asked whether objects (both natural kinds and artifacts) retained their category membership after going through a functional, molecular, or structural change. As would be predicted if function is a core property of artifacts, an artifact described as not being able to perform its function anymore was not considered to continue being the same kind of artifact. For example, a mirror that did not reflect an image was not considered a mirror. Experiment 2 provided even stronger evidence. Subjects were asked to consider three situations: one in which something shared only its structural properties with members of a category, one in which something shared only its molecular structure with members of a category, and one in which something shared only its function with members of a category. Results showed that when the only thing an object shared with category members was its function, it was still considered to be a category member. For example, an object that was not hard and not made of glass but that did reflect an image was considered a mirror. This led the authors to conclude that function is central to category membership.

Keil (1989) also found functions to be central in categories. Subject received variants of common tools. In one condition, these variants looked similar to the original artifact but could not perform the same function (e.g., a hammer with a hole in its head that could not be used to put nails in place). In another condition, the variants looked very different from the standard but their structure still allowed them to perform the same function (e.g., a screwdriver with an unusual handle that could still be used to turn screws). Keil's results revealed that children and adults used function to classify objects and were not misguided by function- irrelevant properties.

In contrast with these two studies, Malt and Johnson (1992) claimed to show that function is neither sufficient nor necessary for categorizing artifacts. Their subjects received descriptions of objects that combined function and physical structure in different ways. In Experiment 1, object descriptions combined a standard function with either the normal physical properties of the object or with unusual physical properties. For example, the standard function of 
a sweater-provides warmth to the upper body when worn over a shirt-was combined either with the standard physical structure of a sweater or with the atypical physical structure of a rubber garment. Independent raters judged the objects with atypical physical structures as able to perform the standard functions (e.g., a rubber garment could still be used to provide warmth to the upper body when worn over a shirt). Nevertheless subjects tended to deny category membership to objects that fulfilled a standard function but that lacked the standard physical structure (e.g., the rubber garment was not considered a sweater). Because possessing a category's standard function did not warrant category membership, Malt and Johnson concluded that function is not sufficient for categorization.

In Malt and Johnson's Experiment 2, objects were described that combined the standard physical structure of an artifact with functions that varied in their degree of resemblance to the standard one (i.e., standard function, related function, bizarre function). For example, the standard function for a boat was transporting people over water, the related function was holding criminals off-shore', the bizarre function was reintroducing marine animals to their habitat. Subjects in this experiment granted category membership to at least half the items that had nonstandard functions (e.g., a boat for holding criminals offshore). Because lacking a category's standard function did not always prevent belonging to a category, Malt and Smith concluded that function is not necessary for category membership.

How might Malt and Johnson's (1992) results be reconciled with the conflicting results of Barton and Komatsu (1989) and Keil (1989)? Why is function not important in the Malt and Johnson studies, but important in the others? We propose that the answer lies in how one thinks about function, either as an isolated unitary property, or as a relational system that links physical structure, function, and background situations (for a related reconciliation of these conflicting results, see Ahn, 1998).

The apparent unimportance of function in Malt and Johnson's results can be explained as the result of their viewing function as an isolated unitary property. Inherent in their experimental designs is the assumption that function is independent of physical structure. According to this logic, the function of an object remains constant as its physical structure varies (e.g., something can function identically as a sweater while made of either wool or rubber). Conversely the structure of an object remains identical as its function varies (e.g., an object used as transportation or as a place to hold criminals offshore has the identical physical structure).

Imagine, though, that subjects don't view function as a unitary property that is independent of physical structure. How might this explain Malt and Johnson's results? In Experiment 1, subjects may have believed that a rubber garment would actually be a poor sweater, even though — in principle - it could function as one. 
If subjects did reason in this manner, then function was not irrelevant to categorization. Instead changes in physical structure, such as rubber vs. wool, may have had significant implications for reasoning about function, which in rum may have affected judgments of category membership. Function only seems insufficient for categorization when one adopts the mistaken assumption that an bject can achieve the full-fledged function of a category independently of its physical structure (e.g., a rubber sweater achieving the full functionality of

a standard sweater). When physical structure is brought to bear on function, however, it's clear that a rubber garment functions poorly as a sweater, thereby making it a poor member of the category. Another important factor is that a rubber garment is also a better member of a competing category, namely, wetsuit, thereby decreasing the garment's membership in sweater.

In Malt and Johnson's Experiment 2, subjects may have similarly reasoned that an object used to hold prisoners offshore could function well as a boat, given that it had the requisite physical properties. Even though the object wasn't being used as a boat, it had the clear potential to be used this way. Indeed it might well function better as a boat than an as offshore prison. If subjects reasoned mis way, then function again determined categorization. Function only appears irrelevant when physical structure and function are viewed function independently e.g., naming something as a boat solely because of its physical structure, while simultaneously believing that its most natural function is as an offshore prison). If, however, subjects reasoned implicitly that the object's most natural function is as a boat - which they probably did - then function controlled categorization.

Viewing function as a relational system further explains why function was mportant for categorization in Barton and Komatsu (1989). In these studies, subjects received a relatively vague description of a single object. In each case, a function was stated for the object (e.g., reflects an image), along with two exclusionary properties about physical structure (e.g., not made of glass and not hard). The negation of two physical properties is essentially an invitation for subjects to infer what the physical properties of the object actually were. Most importantly, subjects had to infer physical properties that would be consistent with the object's stated function. Thus subjects might have reasoned that \& flexible piece of metal with a reflective surface will reflect an image, while being neither hard nor made of glass. If subjects reasoned this way, then they could have easily concluded that the object is indeed a mirror, given that it still achieves a mirror's critical function. Again a complex system of relations underlies the function that controls categorization. Subjects reason about how a particular set of physical properties achieves a particular function. Because the function central for mirror is achieved, an object with atypical physical properties is assigned to the category. Only when the physical structure leads to some other function is membership in the category weak, as for rubber sweater in the Malt and Johnson experiments. 
Finally Keil's (1989) results, too, can be viewed as reflecting a relational system of knowledge about function. In Keil's unusual objects that fulfilled standard functions, the physical structure always afforded the standard function for a category (e.g., a screwdriver), while adding other physical properties that were irrelevant but that didn't affect the object's function. Because subjects could see that the physical structure required for the category's function was present, and because they could see that the unusual physical properties did not compromise this function (e.g., an unusual handle), they concluded that the object was a reasonable category member. Reasoning about category membership again required evaluating a system of relations that integrated structure and function.

Our analysis of these studies suggests that a system of relations linking structure and use represents the function of an object. When this system produces a function consistent with a category, the object is assigned to the category (e.g., Barton \& Komatsu 1989; Keil 1989). When the system produces a function inconsistent with a category, the object is not assigned to it (Malt \& Johnson 1992). Most importantly, function appears central to categorization, and to reflect a complex system of relations-not a unitary property independent of physical structure.

\section{Problem 2: Can Knowledge of Function Support Inductive Inferences?}

One of the primary roles of concepts is to support inductive inference. In the context of categorization research, this means deciding if properties known to be true of one entity can be attributed to other entities for which those properties have not been verified. Assigning an entity to a category is one way to support inductive inference (e.g., Markman 1989; Osherson, Smith, Wilkie, Lopez, \& Shafir 1991; Yamauchi \& Markman 2000). When two objects belong to the same category, people expect that they share important properties. Thus if a novel entity is classified as a bird, people infer that it can fly (even though they may not know this for a fact). Working their way backwards, some researchers have used subjects' willingness to extend attributes from one instance to another as evidence that both are considered members of the same category (e.g., Mak \& Vera 1999; Mandler \& McDonough 1998).

Given the importance of inductive inferences, researchers have asked whether categories based on function support inferences. If functional categories are full strength categories, they should. Much of this discussion has addressed the artifact/ natural kind distinction. Traditionally the inductive potential of artifacts has been thought to be lower than that of natural kinds (e.g., Gelman \& Markman 1986). On this view, a category such as furniture supports less inference than a category 
such as animals. Intuitively, animals share many properties that are not directly perceptible and must be inferred (e.g., their internal structure), whereas items of furniture share only external appearances, which are obvious and need not be inferred.

Results from Gelman (1988, Exp. 1) appeared to support this prediction. Objects at different levels of complexity were used to test 4- and 7-year-olds' inductions with natural kinds and artifacts (object complexity increased with the number of different parts and the amount of complicated internal workings). Subjects learned a new fact about an object and then judged if this fact was true of other objects presented to them. For example, they learned that a carrot is used to make a proboscis, and were later asked whether a test object could perform the same function. Although increasingly complex objects promoted more inferences, natural kinds promoted significantly more inferences than artifacts for 7-year-olds.

Farrar, Raney, and Boyer (1992, Exp. 1) did not replicate these results. When Farrar et al. tested 4-, 7-, and 9-year-old children, they found that object complexity but not object kind (i.e., artifact or natural kind) affected the number of inferences drawn. More complex objects of both types promoted more inferences in 7- and 9-year-olds but not in 4-year-olds, who made more inferences for less complex objects.

Our theme that relational systems underlie function explains this discrepant pattern of results. In Gelman's (1988) procedure, the function of an object typically was novel and had no discernible relation to other object-relevant properties, most notably, physical structure. For example, when used to make a proboscis was presented as the function for carrot, children may not have understood the function, nor how the object's physical properties achieved it. Similar to Malt and Johnson (1992), Gelman treated function as a property that can be ascribed to objects independently of their physical structure. Thus Gelman's (1988) procedure may have produced lower levels of inference because subjects did not understand the relations between physical structure and function. Subjects may have shown stronger inferences for natural kinds than artifacts simply because the former are generally more complex, not because artifacts lack inductive potential.

Conversely, in Farrar et al. (1992), artifacts may have promoted inferences for children because relational knowledge that integrated form and function was available. For example, when told that a computer has a ROM-BIOS inside, children felt warranted to say that a television also has one. If these children had modest relational knowledge about how the insides of electronic appliances cause their behavior, this might have allowed them to generalize from computers to televisions. When children understand the system of relations that underlies an artifact's function, they can reason inductively about its properties. 
A related view on the inductive potential of artifacts incorporates knowledge of the situations in which they are used. Keil (1988) argued that artifact concepts develop in rich socially-based situations. If so, then inferences may draw on the situation in which an artifact is used - not just on its physical structure - thereby making the inductive potential of artifacts at least as high as for natural kinds.

Two studies support Keil's proposal. When inferences are based on the internal properties of objects and ignore the situations in which they are used, functional categories show little inductive potential (Medin, Lynch, Coley, \& Atran 1997). In contrast, when inferences are based on the situations in which artifacts are used, functional categories provide as much inductive potential as natural kinds (Ross \& Murphy 1999). We review these studies in turn.

Medin, Lynch, Coley, and Atran (1997) asked three groups of tree experts to sort trees into categories. Specifically taxonomists, maintenance workers, and landscapers sorted 48 cards for tree names into related groups of trees that go together. Medin et al. used factor analysis on intersubject agreement to examine the experts' sortings. Results showed that around one third of the sorting variance could be explained by morphological and scientific criteria. Additional factors showed that different groups of experts also used criteria related to their respective fields of expertise. For example, landscapers showed functional sortings such as ornamental trees, stand-alone trees, and weed trees. Most importantly, however, landscapers did not use these functional groupings to guide later inductive inferences. Instead they resorted to categories more in line with morphologic and scientific criteria.

These findings might suggest that functional categories such as weed trees are weak in promoting inferences. Other factors, however, qualify this conclusion. Most importantly, Medin et al. asked subjects to make inferences about physiological properties (e.g., reproduction, disease). Perhaps only taxonomic categories warrant physiological inferences. For example, subjects may believe that tree physiology is related to morphology, and therefore believe that classifying trees by morphology is relevant to making inferences about physiological properties. Medin et al.'s results might have differed if the task had asked about functional properties. Perhaps functional categories warrant inferences when the properties are functional.

Ross and Murphy (1999) provide strong support for this conclusion. In Experiments 6 and 7, subjects made inferences about either biochemical or social properties. Examples of biochemical properties are the content of foods, their origin, and macronutrients. Examples of social properties include when foods are eaten, their cost, and their cooking methods. Similar to Medin et al., when subjects made inferences about biochemical properties, they used taxonomic categories to warrant them (e.g., vegetables, meat). Conversely, when subjects made inferences about social properties, they resorted to functional categories (e.g., breakfastfoods, snacks). Heit and Rubenstein (1994) reported similar results. 
As these findings illustrate, categories organized around function can have as much inductive potential as taxonomic categories. When natural kinds appear to promote stronger inferences than artifacts, various confoundings may be involved, such as the match of properties to category types. Another confounding factor is complexity. When the natural kinds used are more complex than the artifacts, this can produce stronger inferences for natural kinds (e.g., when only natural kinds have causally-important internal parts). As the complexity of an object increases, the relational system that explains its operation grows, thereby increasing the potential for inductive inference. When complexity is equated, natural kinds and artifacts may warrant inferences equally.

Natural kinds and artifacts may also differ in the type of relational systems that typically underlie them. Whereas relational systems that link internal parts to external behavior may underlie natural kinds, relational systems that link physical properties to agents and situations may often underlie simple artifacts that lack causally-potent internal parts (see Barsalou, Sloman \& Chaigneau, in press, for an account of these differing systems). A simple artifact does not achieve its function alone but requires coordination with additional entities in the environment (e.g., a hammer requires a nail and a board, both placed in the correct spatial relations by an agent). The relational system for a simple artifact specifies a setting in which its physical structure allows it to perform its function.

Most importantly, inductive inferences may only occur when the relevant relational system is fully in place for a concept, regardless of whether the concept is for a natural kind, complex artifact, or simple artifact. Only when subjects lack full knowledge of the relevant system, or when this knowledge is irrelevant to the property induced, do artifacts fail to exhibit inductive potential.

\section{Problem 3: Can Function Guide Object Naming in Children?}

It seems evident that many objects receive the same name not because of their appearance but because of their function. Take chairs for example. Although chairs vary widely in shape and material, they presumably receive the same name because their function is common (for a more detailed discussion, see Rips 1989). A longstanding debate in the developmental literature has been whether children can use information about function to guide name extensions (i.e., if having the same function motivates them to give the same name to different objects). Two different positions exist. One says that using function to guide categorization does not develop early because it requires subjects to go beyond readily-accessible surface similarities towards relatively-inaccessible relational systems (Gentner \& Rattermann 1991). On this view, young infants focus primarily on properties such as shape and color during categorization (also see Landau, Smith \& Jones 
1998; Smith, Jones \& Landau 1996). The opposing position holds that because children are constantly exposed to function, it should be important in naming from the start (Nelson 1974).

Numerous studies support the first position, especially the conclusion that function does not guide naming until relatively late in development. However the precise age when this ability emerges has been pushed down consistently from study to study. Whereas initial studies put the age limit at around 4 to 5 years (Gentner 1978; Tomikawa \& Dodd 1980), more recent studies report ages as low as 2 (Kemler-Nelson, Russell, Duke \& Jones 2000). As the age limit goes down further, results increasingly favor the position that, from early on, children use information about function when deciding what to call an object.

Why is there this difference in reported age limits? Similar to what we have argued earlier, the age limit has gone down as experimenters have increasingly presented relational information about function to children in an understandable way. In general, it is not enough to show how an object's physical structure is correlated with a certain function. For function to have an effect in categorization, children must understand how the object's physical structure implements its function. They must grasp the full relational system.

In two early studies, the function of novel objects was demonstrated, but no special effort was made to make it comprehensible for children (Gentner 1978; Tomikawa \& Dodd 1980). In Gentner (1978), subjects were familiarized with two novel objects, each with one of two different functions and one of two different physical structures. Later, when subjects were tested with a new object that combined the physical structure of one familiarized object with the function of the other familiarized object, 2- to 5-year olds were likely to extend the name according to physical structure but not to function (note the independent manipulation of structure and function). In contrast, 5- to 9-year-olds were more likely to use function as a basis for categorization. Tomikawa and Dodd (1980) obtained comparable results.

Landau, Smith, and Jones (1998) reported a similar finding. In Experiments 1 and 2, Landau et al. created artifacts that could perform a simple function, such as retrieving small toys or carrying water. Subjects were 2-, 3-, 5-year-olds, and adults. The experimenters either just showed the object or demonstrated its function, while at the same time naming the object. In both experiments, the object's material made the function possible (e.g., a sponge could be used to carry water). Subjects were tested in two tasks: (1) judging if a novel object could perform the original function, and (2) judging if a novel object should be named the same as an earlier one. On the function judgment task, 2-year-olds performed only at chance. Even though 5-year-olds were much better at it, as a group they were still not as accurate as adults. On the naming task, all children (2-, 3-, and 5-year-olds) extended names according to shape. Adults were the only subjects who extended names according to function. 
As already pointed out, a problem with such studies is the assumption that a simple demonstration is sufficient for children to grasp the relational system that underlies an object's function. If the children in these studies had received more support in grasping these systems, the results of these studies might have been quite different. This point becomes more evident when Landau et al.'s (1998) materials are examined. For example, it may have been difficult for young children to understand that being made of sponge allows an object to carry water. Thus younger children's apparent inability here to use function in naming does not necessarily imply that they cannot do so in general. Instead they may have simply lacked an adequate understanding of how an object's material underlies its function. If so, then it also does not follow that younger children solely use physical properties to guide categorization. To the contrary, if these children had understood the demonstrated functions, they might have used them in naming.

Consistent with this argument, other studies show that when young children understand the functions presented to them, they use this knowledge to categorize artifacts. This has been obtained at even younger ages than those tested in the studies examined above. How can a specific function be made more comprehensible for children? Tversky (1989) suggests that object parts coordinate children's ability to link objects and functions. Because parts have both structural and functional aspects, they may play a central role in the relational systems that underlie functional categorization.

Incorporating this principle, Kemler-Nelson (1995) showed that function affects categorization in subjects as young as 3 years of age, using a procedure similar to previous studies, but making the functions comprehensible to children. To maximize the likelihood that children would detect structure-function relations, the functions were made possible by the arrangement of an object's parts. For example, it was clear than an object with several brushes sticking out could only achieve the function of painting when the brushes could distribute paint effectively to a surface. Also, objects' functions were not only demonstrated, but children were allowed to use the objects. Later when children were asked if a set of test objects (shown only in static display) could be called the same name as the standard, children extended the name to new objects that afforded the demonstrated function, not just to objects whose appearance was superficially similar. This same pattern held across different age groups (3-, 4-, and 5-year-olds).

Experiments 2 and 4 in Smith, Jones, and Landau (1996) are also at least partially consistent with the proposal that understanding function is crucial for it to guide categorization. Smith et al. constructed novel artifacts in which a base object was combined with several smaller parts, so that the base could perform one function and the smaller parts could perform a different one (e.g., the base could be used as a telescope, while the parts could be used to hold pens). Subjects (3-year-olds and adults) were familiarized with a novel artifact by demonstration 
of one of its two functions (i.e., the base or part function), and were later allowed to use the object to perform its modeled function. Interestingly, when 3-year- olds where required to categorize new objects, function guided their similarity judgments even when not explicitly requested to do so. Consistent with our theme, children's successful understanding of how parts supported these functions appeared critical. Allowing the children to perform the functions themselves may have also increased the likelihood that they understood the critical part- function relations.

The most definitive support comes from Kemler-Nelson et al. (2000). An object was shown to children while the experimenter named it. The children were then allowed to manipulate the object, and were finally presented with two test objects. The subject's task was to hand the experimenter the test object that should be called the same name as the standard. The most important difference from previous studies was that objects were constructed so that their function was afforded by simple physical principles that 2-yearolds could understand (e.g., when a transparent box that contained beads was turned upside down, the beads would only trickle from one side to the other if the path was unobstructed). Under these conditions function consistently affected 2-year-olds' naming behavior even when no demonstration was performed (only exploration was allowed).

The conclusion from these studies is similar to the one reached for the two research problems described earlier. When structure and function are treated as independent properties, or when the causal relations between structure and function are not clear, function's role is minimized. Function only shows its effect on object naming when meaningful structure-function relations exist, and when subjects understand them. The better both children and adults understand these relations, the more function guides categorization.

\section{Problem 4: Should Function be Understood as Affordances or as Design History?}

The studies reviewed up to this point suggest that knowledge of function reflects the physical interactions that someone has or can potentially have with objects in the course of using them. For example, the function of a chair is its use for sitting. This is probably the most common way in which researchers have thought about function. We call this the affordances view of function, because it can be traced back to Gibson's formulation of affordance theory (Gibson 1977, 1979a, 1979b). On this view, an object's function reflects the actions that can be performed on it, given both its physical structure and the physical structure of the agent interacting with it. 
More recently, however, researchers have argued that understanding the intention of an object's designer is crucial for understanding the object's function (e.g., Bloom 1996, 1998; Gelman \& Bloom 2000; Matan \& Carey 2001; Prasada 1999). In particular, Bloom $(1996,1998)$ assumes that the designer's intention constitutes an artifact's essence (i.e., the intentional theory of function).

The term "essence" has a technical meaning in the philosophy of language, coined in a theory of naming which holds that names are not grounded in mental representations. Instead names are grounded most fundamentally in causal relations to their referents. Kripke (1980), for instance, proposed that proper names and their referents are linked from the moment an initial speaker gives a name to an object. From that moment on, the causal relation between the name and the referent is passed on from speaker to speaker through cultural transmission. A consequence of this account is that naming and conceptualization are completely separate things. The meaning of words (at least for proper names) is not their intension or mental representation but their extension.

Putnam (1975), who coined the term "essence" (i.e., at least as used in this context), proposes that naming and concepts are separate things. He argues that the meaning of words has to do with how things really are in the world, and that people follow this intuition when assigning names to objects. In contrast, when people describe the conceptual aspects of an object (e.g. yellow and sour as properties of lemon), they are simply reporting their knowledge about how the object typically is. Even if this conceptual content varies (e.g., an object is described as lacking the typical properties for its class), people can still use the same name when referring to it. According to Putnam, people follow the intuition that an object's name points to essential aspects of the object class extensionally, and not to a description of its typical appearance. This view has been quite influential in motivating research on people's beliefs about the essences of natural kinds (for an analytical discussion, see Strevens 2000).

Extending this view to artifacts, Bloom $(1996,1998)$ argues that people believe an artifact's essence is whatever the designer intended the artifact to be, not its actual or potential use. For example, an umbrella that is being used as a lampshade would be considered an umbrella and not a lampshade, because that is what its designer intended it to be. According to Bloom, people grasp an object's intended function by performing inference to the best explanation. An object is considered to have function X only if its appearance and potential use are best explained as the result of someone intentionally creating the object to fulfill function $X$. It is worth repeating that the key inferential process is indifferent to the actual use of an object. Instead the theory requires that beliefs about the intentional creation of bjects override their later uses. Although people may lack specific knowledge of an artifact's design history, the intentional theory requires that they have meta-

beliefs about these histories, and that they use these beliefs in categorization. 
In support of this theory, Kemler-Nelson, Frankenfield, Morris, and Blair (2000) report that subjects only consider a function plausible (and use it to guide categorization) if the function provides a plausible causal account of the object's physical structure. If some of the object's physical properties are not explained by the object's function, then the function is not considered plausible. For example, if a stapler is used as a paper holder, a causal account cannot be constructed of how the object was designed, because being a paper holder does not explain several of the stapler's physical properties (e.g., a hinge, staples). As a result, subjects do not perceive being a paper weight to be a plausible function for the object.

Several other studies have also tested this account. In Gelman and Bloom (2000), 3-year-olds, 5-year-olds, and adults were more likely to assign an artifact- name to an object after hearing that the object was created intentionally than after hearing that the object was created accidentally. For example, when subjects learned that a box was created accidentally by a car running over some cardboard, they viewed it as a piece of cardboard, not as a box. Similarly Matan and Carey (2001) found that 6-year-olds and adults (but not 4-year-olds) preferred to name an object according to what it was invented for versus how it was used opportunistically. For example, if an object was created to be a teapot but was then used as a watering can, subjects chose to name it as a teapot. These results further suggest that history plays a central role in how function enters into categorization.

Consistent with our theme, however, the importance of design history in these studies may arise because subjects lack full knowledge of the relational systems that underlie function. As we will illustrate, subjects lacked knowledge of relations important from an affordances point of view. To overcome these omissions, subjects may have used background knowledge about affordances to infer missing relations. In the process, history may have attained an unusual degree of significance.

Consider Gelman and Bloom (2000). These subjects received object descriptions that were clear about object creation but vague about physical structure and use. For example, subjects learned how a box was created accidentally but knew little about its actual physical structure or its actual use as a box. For these accidentally created objects, subjects may have inferred that their physical structure was far from optimal and thus would not fully achieve the required use. An accidentally created box might not seem nearly as well- designed or well-built as a box created intentionally. Furthermore when used as a box, it might seem unlikely to function adequately. Because of the uncertainty surrounding the object's physical structure and use, subjects may have allowed history to dominate their reasoning. They may have assumed that intentional creation led to superior physical structure and functionality than did accidental 
creation. Note however, that history alone is not driving categorization. Instead history coupled with reasoning about physical structure and use is critical.

Similarly in Matan and Carey (2001), subjects received a description of an object's creation and its posterior use but not of its physical structure. For example, subjects heard about a teapot that was used as a watering can but received no information about its physical properties. When an object was created with one intention but used with a different one, subjects may have inferred that its physical structure had to be consistent with the designer's intention. For example, an object created to be a teapot but used as a watering can should have had the physical structure of a teapot. As a result, subjects may have inferred that the physical structure was non-optimal for a watering can. Alternatively if subjects had inferred that the physical structure was adequate, they might not have cared about the object's design history. They might have thought it appropriate to call the object a watering can, even if it had been created for another purpose.

Preliminary work in our laboratory supports these reinterpretations of the findings in Gelman and Bloom (2000) and Matan and Carey (2001). When adequate information about physical structure and actual use is provided, an object's history is not nearly as central as the studies above suggest. In Chaigneau (2002), subjects received scenarios that described both an object's design history and its actual use. In some scenarios, the creation and use of an object was accidental, but the necessary structure and actions that afforded its function were present. For example, subjects believed that an object having the structure of a mop and used as a mop was a good mop, even when it had been created accidentally, and even when the agent did not intend to use it as a mop. In other scenarios, the creation and use of a mop were fully intentional, but the required physical structure and actions were lacking. For example, an object was intended to be created and used as a mop, but its physical structure was inadequate. Under these conditions, subjects did not perceive these objects to be good mops.

Across the experiments in Chaigneau (2002), physical affordances dominated history when subjects reasoned about artifact categories and their functions (although history did play a minor role). These results question the claim that design histories constitute the essences of artifacts. Instead these results suggest that affordances are much more central.

Nevertheless the results of Kemler-Nelson et al. (2000), Gelman and Bloom (2000), and Matan and Carey (2001) all show that history can become important under certain conditions. Notably, however, these are relatively unusual conditions when an object's parts are unexplained, or when information about an object's physical structure or actual use are missing. Under these conditions, history can become important, although the reasoning about history is always closely entwined with reasoning about physical structure and use. Again entire relational systems underlie knowledge of function. What the studies in this section add to our story 
is that these systems include knowledge of design history, not just knowledge of affordances.

\section{Conclusion}

The account of function that emerges here is not of a simple and unitary property. Rather function appears to be a relational system that links entities, events, and situations. Throughout this article, we have presented evidence that supports this view. When subjects know the relational systems that underlie function, they use it to categorize, to name, to guide inferences, and to fill gaps in knowledge. As these literatures show, many different relations must be known to understand function. Most importantly, only when subjects fully understand the relevant system of relations does function show its substantial effects on categorization. Conversely, when the full system of relations is not known, function is relegated to a peripheral role. In particular, when function is presented independently of structure, or when the structure-function relation is not clear, the role of function declines (understandably).

It is also apparent, however, that function does not depend completely on an object's affordances. An object's design history is also important. Although history may not be as central as some researchers believe, it is nevertheless important in mature conceptualizations of function. History clearly enters into to how people reason about the function of objects, especially under conditions when information relevant to understanding affordances is lacking.

Our view of function as a relational system has directed our research into detailed analyses of this knowledge. Viewing function as relational has forced us to consider explicitly what concepts and relations underlie these systems. As a result, we have developed the HIPE theory of function, which attempts to lay out this conceptual structure in some detail (Barsalou et al., in press). We are also testing some of the theory's predictions, and hope that these studies will increase our understanding of function's role in categorization (Chaigneau 2002; Chaigneau, Barsalou \& Zamani 2002).

\section{References}

Ahn, W. (1998). Why are different features central for natural kinds and artifacts? The role of causal status in determining feature centrality. Cognition, 69(2), 135-178.

Barsalou, L.W. (1992). Frames, concepts, and conceptual fields. In E. Kittay \& A. Lehrer (Eds.), Frames, fields, and contrasts: New essays in semantic and lexical organization (21-74). Hillsdale, NJ: Lawrence Erlbaum Associates. 
Barsalou, L. W., Sloman, S. A., \& Chaigneau, S. E. In press. The HIPE theory of function. To appear in L. A. Carlson \& E. van der Zee (Eds.), Functional features in language and space: Insights from perception, categorization and development. Oxford: Oxford University Press.

Barton, M. E. \& Komatsu, L. K. (1989). Defining features of natural kinds and artifacts. Journal of Psycholinguistic Research, 18(5), 433-447.

Bloom, P. (1996). Intention, history, and artifact concepts. Cognition, 60, 1-29.

Bloom, P. (1998). Theories of artifact categorization. Cognition, 66, 87-93.

Chaigneau, S. E. (2002). Studies in the conceptual structure of object function. Dissertation in progress, Emory University, Atlanta, GA.

Chaigneau, S. E., Barsalou, L. W., \& Zamani, M. (2002). Function as a multimodal relational construct. Manuscript in preparation.

Estes, W. K. (1986). Array models for category learning. Cognitive Psychology, 18, 500- -548.

Farrar, M. J., Raney, G. E., \& Boyer, M. E. (1992). Knowledge, concepts, and inferences in childhood. Child Development, 63, 673-691.

Gelman, S. A. (1988). The development of induction within natural kind and artifact categories. Cognitive Psychology, 20, 65-95.

Gelman, S. A., \& Bloom, P. (2000). Young children are sensitive to how an object was created when deciding what to name it. Cognition, 76, 91-103.

Gelman, S. A., \& Markman, E. M. (1986). Categories and induction in young children. Cognition, 23(3), 183-209.

Gentner, D. (1978). What looks like a jiggy but acts like a zimbo? A study of early word meaning using artificial objects. Papers and Reports on Child Language Development, 15, 1-6.

Gentner, D., \& Markman, A.B. (1997). Structure mapping in analogy and similarity. American Psychologist, 52, 45-56.

Gentner, D. \& Rattermann, M. I. (1991). Language and the career of similarity. In S. Gelman \& J. Byrnes (Eds.), Perspectives on language and thought: Interrelations in development (pp. 225-277). New York, NY: Cambridge University Press.

Gibson, I. J. (1977). The theory of affordances. In R. Shaw \& I. Bransford (Eds.), Perceiving, acting, and knowing: toward an ecological psychology. Hillsdale, NI: Erlbaum.

Gibson, J. J. (1979a). The ecological approach to visual perception. Boston: Hughton- Mifflin.

Gibson, J. I. (1979b). The perception of the visual world. Boston: Hughton-Mifflin. Hampton,

J. A. (1979). Polymorphous concepts in semantic memory. Journal of Verbal

Learning \& Verbal Behavior, 18(4), 441-461.

Hampton, J. A. (1995). Testing the prototype theory of concepts. Journal of Memory \& Language, 34(5), 686-708.

Heit, E. (1992). Categorization using chains of examples. Cognitive Psychology, 24, 341-380.

Heit, E., \& Rubinstein, J. (1994). Similarity and property effects in inductive reasoning. Journal of Experimental Psychology: Learning, Memory, \& Cognition, 20(2), 411- -422. 
Hintzman, D. L. (1986). Schema abstraction in a multiple-trace memory model. Psychological Review, 93, 411-428.

Holyoak, K.J., \& Thagard, P. (1997). The analogical mind. American Psychologist, 52, 35-44.

Keil, F. C. (1988). Commentary: Conceptual heterogeneity versus developmental homogeneity (on chairs and bears and other such pairs). Human Development, 31, 35-43.

Keil, F. C. (1989). Concepts, kinds, and cognitive development. Cambridge, MA: MIT Press.

Kemler-Nelson, D. G. (1995). Principle-based inferences in young children's categorization: Revisiting the impact of function on the naming of artifacts. Cognitive Development, 10, 347-380.

Kemler-Nelson D.G., Frankenfield, A., Morris, C., \& Blair, E. (2000). Young children's use of functional information to categorize artifacts: three factors that matter. Cognition, 77(77), 133-168.

Kemler-Nelson, D. G., Russell, R., Duke, N., \& Jones, K. (2000). Two-year-olds name artifacts by their functions. Child Development, 77(5), 1271-1288.

Kripke, S. A. (1980). Naming and Necessity. Oxford, UK: Basil Blackwell.

Landau, B., Smith, L. B., \& Jones, S. S. (1998). Object shape, object function, and object name. Journal of Memory and Language, 38, 1-27.

Mak, B. S., \& Vera, A. H. (1999). The role of motion in children's categorization of objects. Cognition, 71, B11-B21.

Malt, B. C, \& Johnson, E. C. (1992). Do artifact concepts have cores? Journal of Memory and Language, 31, 195-217.

Mandler, J. M., \& McDonough, L. (1998). Studies in inductive inference in infancy. Cognitive Psychology, 37, 60-96.

Markman, E. M. (1989). Categorization and naming in children. Cambridge, MA: The MIT Press.

Markman, A.B., \& Gentner, D. (1997). The effects of alignability on memory. Psychological Science, 8, 363-367.

Matan, A., \& Carey, S. (2001). Developmental changes within the core of artifact concepts. Cognition, 78, 1-26.

Medin, D. L, Lynch, E. B., Coley, J. D., \& Atran, S. (1997). Categorization and reasoning among tree experts: Do all roads lead to Rome? Cognitive Psychology, 32, 49-96.

Medin, D. L. \& Ortony, A. (1989). Psychological essentialism. In S. Vosniadou \& A. Ortony (Eds.), Similarity and analogical reasoning (pp. 179-195). New York: Cambridge University Press.

Medin, D. L. \& Schaffer, M. M. (1978). Context theory of classification learning. Psychological Review, 85, 207-238.

Nelson, K. (1974). Concept, word, and sentence: Interrelations in acquisition and development. Psychological Review, 87(4), 267-285.

Nosofsky, R. M. (1986). Attention, similarity and the identification-categorization relationship. Journal of Experimental Psychology: General, 115, 39-57. 
Nosofsky, R. M. (1991). Exemplars, prototypes and similarity rules. In A. Healey, S. Kosslyn, \& R. Shiffrin (Eds.), From learning theory to connectionist theory: Essays in honor of W K. Estes, Vol. 1 (pp. 149-168). Hillsdale, NJ: Erlbaum.

Osherson, D., Smith, E. E., Wilkie, O., Lopez, A., \& Shafir, E. (1991). Category-based induction. Psychological Review, 97, 185-200.

Pearl, J. (2000). Causality: Models, reasoning, and inference. Cambridge, UK: Cambridge University Press.

Prasada, S. (1999). Names for things and stuff: An Aristotelian perspective. In R. Jackendoff, P. Bloom, \& K. Wynn (Eds.), Language, logic, and concepts: Essays in honor of John Macnamara (pp. 119-146). Cambridge, MA: MIT Press.

Putnam, H. (1975). The meaning of "meaning". In H. Putnam (Ed.), Mind, Language and Reality. Philosophical Papers, Vol. 2. Cambridge, UK: Cambridge University Press.

Rips, L. J. (1989). Similarity, typicality, and categorization. In S. Vosniadou \& A. Ortony (Eds.), Similarity and Analogical Reasoning (pp. 21-59). New York: Cambridge University Press.

Rosch, E. (1975). Cognitive representation of semantic categories. Journal of Experimental Psychology: General, 104(3), 192-233.

Rosch, E., \& Mervis, C. B. (1975). Family resemblances: Studies in the internal structure of categories. Cognitive Psychology, 7(4), 573-605.

Rosch, E., Simpson, C., \& Miller, R. S. (1976). Structural bases of typicality effects. Journal of Experimental Psychology: Human Perception and Performance, 2(4), 491-502.

Ross, B. H., \& Murphy, G. L. (1999). Food for thought: Cross-classification and category organization in a complex real-world domain. Cognitive Psychology, 38, 495-553.

Sloman, S. A., Love, B, \& Ahn. W. (1998). Feature centrality and conceptual coherence. Cognitive Science, 22, 189-228.

Smith, E. E., \& Medin, D. L. (1981). Categories and concepts. Cambridge, MA: Harvard University Press.

Smith, E. E., \& Sloman, S. A. (1994). Similarity- versus rule-based categorization. Memory \& Cognition, 22, 377-386.

Smith, Li B,, Jones, S. S., \& Landau, B. (1996). Naming in young children: a dumb attentional mechanism? Cognition, 60, 143-171.

Strevens, M. (2000). The essentialist aspect of naive theories. Cognition 74, 149-175.

Tomikawa, S. A. \& Dodd, D. H. (1980). Early word meanings: Perceptually or functionally based. Child Development, 51, 1103-1109.

Tversky, B. (1989). Parts, partonomies, and taxonomies. Developmental Psychology, 25, $983-$ 995.

Wright, L. (1973). Functions. Philosophical Review, 82, 139-168.

Yamauchi, T. \& Markman, A. B. (2000). Inference using categories. Journal of Experimental Psychology: Learning, Memory, \& Cognition, 26(3), 776-795. 